\title{
Les unités de mesure dans les documents officiels du dix-neuvième siècle au Bas-Canada et au Québec
}

\section{Régis Thibeault}

Volume 43, numéro 2, automne 1989

URI : https://id.erudit.org/iderudit/304788ar

DOI : https://doi.org/10.7202/304788ar

Aller au sommaire du numéro

Éditeur(s)

Institut d'histoire de l'Amérique française

ISSN

0035-2357 (imprimé)

1492-1383 (numérique)

Découvrir la revue

Citer cette note

Thibeault, R. (1989). Les unités de mesure dans les documents officiels du dix-neuvième siècle au Bas-Canada et au Québec. Revue d'histoire de l'Amérique française, 43(2), 221-232. https://doi.org/10.7202/304788ar

\section{Résumé de l'article}

L'utilisation des données colligées dans plusieurs séries de documents, en particulier dans les recensements publiés du XIX ${ }^{\mathrm{e}}$ siècle, s'accompagne de diverses contraintes méthodologiques. Un des principaux biais observé, en rapport avec les divisions de recensement du Québec, provient de l'usage simultané des systèmes de mesure français et anglais pour la désignation des unités de volume et de superficie, sans même que cette pratique ne soit spécifiquement mentionnée. Ce problème doit d'abord être posé en référence avec certains paramètres de l'occupation du sol et des institutions qui s'y rattachent. Ainsi, on reconnaît généralement la préséance des mesures françaises (l'arpent et le minot) dans le Québec seigneurial alors que dans les nouvelles divisions cantonales l'acre et le boisseau seraient les mesures utilisées. Ces repères ne sont toutefois qu'indicatifs. En effet, dans certaines régions comme le Saguenay, l'ambiguïté se complique du fait que la division initiale de la terre se fait sur une base cantonale alors que le peuplement origine presque exclusivement des zones seigneuriales. Dans ce cas, et sans autre validation, il est difficile de savoir à quel système de mesure on a affaire. En conséquence, nous croyons que tout modèle de conversion des unités de mesure devrait nécessairement tenir compte de ce problème de méthode qui affecte sans doute plus d'une région du Québec.
Tous droits réservés @ Institut d'histoire de l'Amérique française, 1989

Ce document est protégé par la loi sur le droit d'auteur. L'utilisation des services d'Érudit (y compris la reproduction) est assujettie à sa politique d'utilisation que vous pouvez consulter en ligne.

https://apropos.erudit.org/fr/usagers/politique-dutilisation/ 


\title{
NOTE DE RECHERCHE
}

\section{LES UNITÉS DE MESURE DANS LES DOCUMENTS OFFICIELS DU DIX-NEUVIÈME SIÈCLE AU BAS-CANADA ET AU QUÉBEC}

\author{
RÉGIS THIBEAULT \\ (SOREP) \\ Université du Québec à Chicoutimi
}

\begin{abstract}
RÉSUMÉ
L'utilisation des données colligées dans plusieurs séries de documents, en particulier dans les recensements publiés du XIXe siècle, s'accompagne de diverses contraintes méthodologiques. Un des principaux biais observé, en rapport avec les divisions de recensement du Québec, provient de l'usage simultané des systèmes de mesure français et anglais pour la désignation des unités de volume et de superficie, sans même que cette pratique ne soit spécifiquement mentionnée. $\mathrm{Ce}$ problème doit d'abord être posé en référence avec certains paramètres de l'occupation du sol et des institutions qui s'y rattachent. Ainsi, on reconnaît généralement la préséance des mesures françaises (l'arpent et le minot) dans le Québec seigneurial alors que dans les nouvelles divisions cantonales l'acre et le boisseau seraient les mesures utilisées. Ces repères ne sont toutefois qu'indicatifs. En effet, dans certaines régions comme le Saguenay, l'ambiguité se complique du fait que la division initiale de la terre se fait sur une base cantonale alors que le peuplement origine presque exclusivement des zones seigneuriales. Dans ce cas, et sans autre validation, il est difficile de savoir à quel système de mesure on a affaire. En conséquence, nous croyons que tout modèle de conversion des unités de mesure devrait nécessairement tenir compte de ce problème de méthode qui affecte sans doute plus d'une région du Québec.
\end{abstract}

\begin{abstract}
The use of data collected from several series of documents, particularly census published in the 19th century, is marred by a variety of methodological constraints. One of the principal biases observed with reference to the Québec census divisions results from the simultaneous use of French and English measuring systems for designating units of volume and surface with no explicit reference to which system is being utilized. The problem becomes particularly evident when dealing with certain parameters relating to the occupation of the land and associated institutions. In general, there was a prevalence of French measurements (the arpent and the minot) in seigneurial Québec, whereas in the new cantonal divisions, the acre and the bushel were the measurements most often used. This general guideline is indicative, however. In fact, in certain regions such as the Saguenay, the uncertainty is heightened by the fact that the original division of the land was performed on a cantonal basis, whereas the settlers came almost exclusively from the seigneurial zones. In such cases, in the absence of further validation, it is difficult to know which system of measurement was actually used. Consequently, we believe that any model for converting units of measurement must take into account this methodological problem, which doubtless affects more than one region in Québec.
\end{abstract}

RHAF, vol. 43, no 2, automne 1989 


\section{INTRODUCTION}

Dans une perspective historique, les unités de mesure contenues dans plusieurs séries de documents au Québec posent un problème méthodologique fondamental autant pour l'utilisateur que pour le gestionnaire de la source en question. Depuis la conquête anglaise de 1760 , nous devons en effet concilier l'usage simultané des systèmes de mesure britannique et français, sans qu'il soit toujours possible de distinguer immédiatement lequel des deux systèmes est appliqué. Cette ambiguïté peut engendrer des biais importants non seulement sur le plan de l'interprétation et de l'analyse des documents concernés (par exemple en ce qui a trait aux mesures longitudinales ou comparatives des productions, des rendements, etc.) mais aussi sur le plan de la description de leur contenu. En outre, c'est un problème présent dans plusieurs types de sources actuellement disponibles et largement consultées (archives publiques, journaux, inventaires, recensements, etc.). Dans tous ces cas, en l'absence de références pertinentes, le chercheur se demande s'il doit convertir ou non les données qui lui sont fournies. Nous proposons ici certains éléments de solution à ce problème $e^{1}$.

\section{ÉTAT DE LA QUESTION}

Une des principales sources utilisées par les historiens et qui se voit directement confrontée par cette question est sans doute les recensements manuscrits et publiés du gouvernement canadien. Nous nous référons en particulier aux données agraires colligées dans les cinq recensements du dix-neuvième siècle ${ }^{2}$. Déjà, des travaux de certains chercheurs portant sur l'agriculture québécoise et plus largement sur la société rurale du siècle passé ont révélé le problème posé par l'utilisation des recensements canadiens ${ }^{3}$. Parallèlement, d'autres travaux étaient exclusivement orientés vers une critique de contenu de recensements décennaux ${ }^{4}$. Une préoccupation commune à ces recherches est l'ambi-

1 Ce texte s'inscrit dans le prolongement d'un mémoire de maîtrise déposé au printemps 1988 à l'Université du Québec à Chicoutimi, dans le cadre du programme de maîtrise en études régionales. Régis Thibeault, Les transformations de l'agriculture au Saguenay (1852-1971). Il a bénéficié des nombreux conseils de Gérard Bouchard, directeur du mémoire, et de Normand Séguin, ainsi que du soutien technique et financier du Centre interuniversitaire de recherches sur les populations (SOREP).

${ }_{2}$ Recensements de 1852 vol. II, 1861 vol. II, 1871 vol. III, 1881 vol. III et 1891 vol. IV.

3 Normand Séguin, «L'agriculture de la Mauricie et du Québec, 1850-1950», Revue d'histoire de l'Amérique française, 35,4 (mars 1982): 537-563; Gérard Bouchard et Régis Thibeault, "L'économie agraire et la reproduction sociale dans les campagnes saguenayennes», Histoire sociale/Social History, 18,36 (novembre 1986): 237-259; Normand Séguin, René Hardy et Louise Verreault-Roy, L'agriculture en Mauricie; dossier statistique 1850-1950 (Trois-Rivières, Université du Québec à Trois-Rivières), 175 p.; Frank Lewis et Marvin McInnis, «The Efficiency of the French-Canadian Farmer in the Nineteenth Century», The Journal of Economic History, 11,3 (Sept. 1980): 497-514.

4 Normand Fortier, «Les recensements canadiens et l'étude de l'agriculture québécoise, 1852-1901», Histoire sociale/Social History, 17,34 (novembre 1984): 257-286. Aussi, Marvin McInnis, «Some Pitfalls in the 1851-1852 Census of Agriculture of Lower Canada», Histoire sociale/Social History, 14,27 (mai 1981): 219-231. 
guité causée par l'utilisation dans un même espace et à un même moment de deux systèmes de mesure associés à des modes distincts de tenure de la terre. Avec le système de mesure anglais, les superficies et les volumes sont désignés respectivement par l'acre et le boisseau, par opposition à l'arpent et au minot français. Bien que, dans les recensements publiés, l'acre et le boisseau soient toujours mentionnés comme unités de mesure (sauf en 1861 où les volumes sont exprimés en minot), l'arpent et le minot seraient les mesures en usage dans la plupart des districts du Québec, c'est-à-dire dans l'ancienne zone d'occupation seigneuriale. Par ailleurs, dans les cantons ou «townships», les unités de mesure anglaises seraient celles en usage ${ }^{5}$. En réalité, les frontières qui marquent la préséance d'un système sur l'autre ne sont pas bien connues ${ }^{6}$. Un tel découpage, comme nous le verrons plus loin, fait fi d'une réalité beaucoup plus complexe sur les marges des aires géographiques où les deux systèmes, loin d'être en opposition, s'interpénètrent. Du même coup, cette coexistence empêche toute conversion unilatérale d'un système à l'autre applicable à l'ensemble des districts de la province'

\section{L'EXEMPLE SAGUENAYEN}

Les unités de mesure en usage au Saguenay au dix-neuvième siècle sont soumises à une double logique: d'une part, à celle de l'origine du peuplement et, d'autre part, à celle du mode de concession des terres. La population pionnière de la région provenait très majoritairement de Charlevoix ${ }^{8}$. C'est une population dont on peut penser qu'elle hérite des pratiques associées à la tenure seigneuriale, donc du système de mesure qui lui est tributaire. Toutefois, l'ouverture du Saguenay à la colonisation repose sur un mode cantonal d'occupation; la division des terres se fait donc en acres. Or, les recensements manuscrits saguenayens s'expriment en arpents et en minots, tandis que les recensements

5 L'importance des écarts entre les unités de mesure françaises et anglaises commande l'établissement d'un facteur de normalisation. Un arpent équivaut à 0,845 acre alors qu'un minot peut égaler de 1073 à 1107 boisseau, selon qu'il s'agit du boisseau de Winchester ou du boisseau Imperial. Recensement de 1901, vol. II; F. Lewis et R. M. McInnis, "The Efficiency of the French-Canadian Farmer in the Nineteenth Century», The Journal of Economic History, 11,3 (Sept. 1980): 497-514. L'ampleur de la variation entre les deux échelles de mesure est accentuée par le fait qu'en plus de la différence linéaire de l'arpent à l'acre et du minot au boisseau, les deux écarts s'additionnent dans le cas d'une mesure de rendement. En effet, dans une mesure de productivité, le nombre de boisseaux à l'acre est supérieur de près de trente pour cent au nombre de minots à l'arpent.

6 Normand Fortier, loc. cit., 270.

7 Une comparaison entre les recensements manuscrits et publiés de 1852, 1861 et 1871 indique que seules les données contenues dans le recensement de 1871 ont été converties en mesures anglaises. Rappelons que, à partir de 1881, les manuscrits des recensements agricoles ne sont plus disponibles. Toutefois, $\mathrm{N}$. Fortier parle d'une similitude évidente entre ce recensement et celui de 1871. Théoriquement, le travail de conversion resterait à faire en 1852, 1861 et très certainement en 1891 .

8 Gérard Bouchard, «Le peuplement blanc», Christian Pouyez et al., Les Saguenayens. Introduction à l'histoire de la population du Saguenay, XVIe-XXe siècles (Québec, Presses de l'Université du Québec, 1983), 396 p. Voir chapitre 4. 
publiés s'expriment en acres et en boisseaux. On voit que l'élaboration d'un modèle de conversion des unités de mesure doit nécessairement tenir compte de ce double caractère inhérent à l'occupation du territoire (origine du peuplement et division initiale de la terre).

Notre démonstration repose tout d'abord sur une confrontation entre les données manuscrites et les données publiées des recensements. C'est ce que nous avons fait à partir d'un échantillon de districts saguenayens, pour un certain nombre de productions ${ }^{9}$. Pour les deux permiers recensements (1852 et 1861), il appert que du manuscrit au volume publié, aucune conversion n'a été faite. On retrouve en effet les mêmes nombres, même si dans le premier cas on se réfère aux unités françaises et dans l'autre aux unités anglaises. Par ailleurs, en 1871, les responsables du recensement auraient effectivement procédé à une conversion des données avant de les publier. Théoriquement, il resterait donc à convertir celles des recensements publiés de 1852 , 1861 et $1891^{10}$.

Le relevé des données agricoles des recensements du dix-neuvième siècle a donc été le même au Saguenay que celui observé par Normand Fortier ${ }^{11}$ pour l'ensemble du Québec. Cependant, la pertinence des choix effectués par les recenseurs d'une décennie à l'autre est loin d'être établie, tout au moins dans le cas du Saguenay. C'est ce que nous avons voulu vérifier en confrontant aux relevés des recenseurs d'autres sources totalement indépendantes.

\section{LES UNITES DE SUPERFICIE ${ }^{12}$}

À partir d'une liste des concessions de terres de la Couronne ${ }^{13}$, nous avons relevé en détail les superficies concédées dans tous les cantons saguenayens. Il appert que toutes les concessions ont été faites en acres. Cette première vérification suggère que certaines superficies dans

9 Pour chacun des cantons (townships) Laterrière, Chicoutimi, Jonquière et Métabetchouan, nous avons confronté cinq unités comparatives. Trois réferent à des superficies (en culture, ensemencée en blé, totale occupée) et deux à des volumes (productions de blé et d'avoine).

10 Nous avons déjà indiqué que le recensement de 1881 a sans doute été fait de la même manière que le précédent. Aussi nous présumons que les données qui y sont contenues ont préalablement été converties en unités anglaises, même s'il n'existe pas de manuscrits pour l'attester. En 1891, il n'existe pas non plus de manuscrits disponibles. Cependant, une note dans le recensement de 1901 indique, en se référant au recensement de 1891: «Le travail de compilation a été fait par le personnel employé au bureau du recensement à Ottawa; mais malheureusement on a négligé de faire la réduction des arpents en acres et des minots en boisseaux.» (Recensement canadien, 1901, volume II, introduction).

11 Normand Fortier, loc. cit..

12 Précisons que la démonstration des mesures en usage pose des problèmes très différents selon qu'il s'agit d'unités de superficie ou de volume de production. Le travail de validation doit reposer sur des sources tout à fait différentes. Pour cette raison, nous aborderons séparément chacune de ces deux questions.

13 Voir J.-C. Langelier, Liste des terrains concédés par la Couronne dans la province de Québec. De 1763 au 31 décembre 1890 (Québec, Législature du Québec, 1891), 1921 p. L'auteur y dresse la liste des terres concédées de 1763 à 1890. Les concessions sont regroupées par canton. On y retrouve le numéro des lots, le rang, la superficie concédée, la date d'émission des lettres patentes et la référence au livre d'enregistrement. 
les recensements manuscrits ont pu être déclarées en acres, principalement dans le cas de la superficie totale occupée. Celle-ci devrait donc théoriquement correspondre à la superficie de la terre concédée. En d'autres mots, nous n'aurions pas à convertir les superficies déclarées dans les recensements de 1852, 1861 et 1891, du moins pour le Saguenay, contrairement à la présomption évoquée plus haut.

Toutefois, d'autres facteurs ont pu jouer lors de la déclaration des unités de mesure dans les recensements. L'occupant pouvait tout aussi bien déclarer en arpents des superficies qui lui avaient été concédées en acres. En outre, le cas des «squatters», appparemment nombreux à cette époque au Saguenay représente un autre élément d'incertitude ${ }^{14}$. Enfin, même si la superficie occupée avait été déclarée en acres (parce que c'était la mesure en usage lors de la concession), les autres superficies qui la composaient (en culture, en pâturages, etc.) auraient pu tout aussi bien l'être en arpents. Partant du principe que le système de concession des terres s'exprime originellement en acres, le fait qu'il soit exprimé en arpents dans les recensements manuscrits est dû soit à une conversion, soit à un abus de langage. Nous avons tenté d'éclairer la question au moyen de deux types de comparaison effectués à partir des concessions de terres et des trois recensements nominatifs disponibles pour le dix-neuvième siècle.

Nous servant d'abord de la Liste des terres concédées par la Couronne ${ }^{15}$, nous avons calculé pour certains cantons saguenayens la proportion de lots dont la superficie correspond à un multiple de 50 acres (ou à un demi-lot). Les superficies inférieures ont donc volontairement été exclues. Un comptage similaire a été fait à partir des superficies déclarées dans les recensements manuscrits. Il restait à comparer pour les périodes correspondantes le pourcentage de lots de chacune des deux sources pour lesquelles la superficie est divisible par $50^{16}$. Prenons en exemple le canton Laterrière au recensement de 1852. Langelier y dénombre 17 concessions de terre avant 1853 et toutes ont une superficie qui correspond à un multiple de 50 acres. Dans le manuscrit de 1852 , on recense à Laterrière 57 occupants de terre, dont $55(95 \%)$ déclarent une superficie divisible par ce même multiple. Nous sommes donc en droit de penser que les superficies du canton Laterrière, dans le recensement de 1852, sont bel et bien déclarées en acres, en dépit du fait que l'on indique «arpents» dans le recensement manuscrit. Une

14 Daniel Larouche, «Le mouvement de concession des terres à Laterrière», Normand Séguin, dir., Agriculture et colonisation au Québec (Montréal, Boréal Express, 1983), 220 p. Voir page 175 .

15 Jean-C. Langelier, Liste des terrains concédés par la Couronne dans la province de Québec. De 1763 au 31 décembre 1890 (Québec, Législature du Québec, 1891), 1921 p.

${ }_{16}$ Cette méthode de comparaison a été utilisée, entre autres, par Normand Fortier pour attester la décision des recenseurs relativement aux superficies déclarées dans les cantons d'Upton et d'Acton, dans l'Estrie. 
division initiale de la terre en multiples de 50 acres (100\%) ne saurait devenir à 95\% un morcellement divisible par 42 acres (ou 50 arpents). En outre, il n'y a même pas deux ans d'écart entre la première concession à Laterrière (21 octobre 1850$)$ et la date du recensement ( 12 janvier 1852). On ne peut non plus arguer que seule la superficie totale occupée aurait pu être donnée en acres comme nous l'évoquions précédemment, alors que l'ensemble des autres mesures de superficie serait donnée en arpents. En effet, dans le manuscrit de 1852, comme dans le volume publié d'ailleurs, la somme de la superficie «ayant produit une récolte en 1851» (ou superficie ensemencée) et des pâturages correspond à la superficie en culture ${ }^{17}$. L'addition de cette dernière à la superficie «terre en bois debout et inculte» donne exactement la superficie «occupée» dans le manuscrit ou «possédée» dans le volume publié. Cette équation semble indiquer que non seulement la superficie totale occupée mais bien l'ensemble des aires qui la composent sont véritablement des estimations en acres. Une vérification serrée au niveau de chacune des superficies mises en culture nous aurait donné une plus grande assurance puisqu'elles reflètent directement l'utilisation de la terre. Toutefois, pour un certain nombre de productions, seules sont recensées les quantités récoltées. C'est le cas en particulier du foin et de certaines autres productions plus marginales (trèfle, houblon, fèves, etc.). Il nous était par ailleurs impossible d'évaluer les superficies de ces récoltes par une mesure de rendement moyen. D'abord, il peut être aléatoire d'extrapoler à partir d'un autre recensement, la productivité en agriculture étant très variable. Enfin, la principale production pour laquelle nous n'avons pas la superficie est le foin. Les volumes de foin récoltés cumulent deux mesures (les bottes et les tonneaux), sans qu'il ne soit possible de départager les valeurs attribuables à l'une et à l'autre.

Cependant, pour au moins un canton de la région dans le recensement de 1852 (le canton Kénogami), le total de la production se compose de récoltes dont les superficies ont été recensées. Il s'agit essentiellement de 13 acres d'avoine et de 2 acres de pois. Si, par ailleurs, il s'agissait d'arpents, ce total serait ramené à 13 acres $(12,6)$. Mais le total de la superficie cultivée dans le canton Kénogami en 1852 est bien de 15 acres. En y ajoutant la «terre en bois debout» de 235 acres (il n'y a pas de pâturage déclaré), nous arrivons à un total de 250 acres, le même que la superficie possédée. L'exemple du canton Kénogami est bien sûr un cas particulier où les superficies ensemencées sont restreintes, mais il renforce nos présomptions, à savoir que toutes les superficies contenues dans le recensement de 1852 au Saguenay sont données en acres.

17 La «superficie en culture» est en fait une mauvaise appellation de la superficie améliorée ou une traduction erronée de «improved land». 
La comparaison du nombre de terres divisibles par un multiple de 50 acres dans la liste des terres concédées et dans les données des recensements conduit à des conclusions similaires en 1861 et en $1871^{18}$. Seul fait exception le canton Labarre en 1871 où le nombre de terres divisibles par 50 acres est de $75 \%$ sur la liste des terres concédées et de $12 \%$ dans le recensement. Toutefois, dans plusieurs cas, les lots dont la superficie n'est pas divisible par 50 acres ne s'approchent pas plus de la mesure équivalente en arpents ( 42 acres). En conséquence, l'usage des mesures anglaises serait généralisé à l'échelle de la région, du moins pour la désignation des unités de superficies.

À l'aide du recensement manuscrit de 1871 et de la liste des terres concédées par la Couronne, nous voulions étayer nos conclusions précédentes par une autre approche comparative mais cette fois à une échelle non agrégée. À l'aide d'un échantillon d'occupants de terres (répartis dans les cantons Laterrière, Jonquière et Hébertville), nous avons comparé les superficies déclarées dans le recensement avec celles effectivement concédées par le gouvernement. Par exemple, à Laterrière, sur 70 occupants relevés dans le recensement de 1871, cing ont été retrouvés sur la liste des terres concédées par la Couronne ${ }^{19}$. De ce nombre, trois déclarent une superficie possédée (dans le recensement) identique à celle qui a été concédée alors que les deux autres cas ne s'approchent ni de la superficie en acres ni de son équivalent en arpents. Les proportions sont à peu près les mêmes dans les autres cantons de l'échantillon. Ces résultats sont donc conformes à nos observations antérieures.

En résumé, s'agissant du Saguenay, nous croyons plus sûr de conserver comme telles les superficies déclarées dans les recensements de 1852,1861 et 1891 , et de reconvertir en acres celles apparaissant dans les recensements de 1871 et 1881 . En fait, pour ces deux années, on reproduit les valeurs des recensements manuscrits.

18 En 1861 , la comparaison a porté sur les cantons Laterrière, Jonquière, Tremblay et Labarre et, en 1871, sur Laterrière, Jonquière, Labarre et Caron. Aussi, voir le détail des résultats dans Régis Thibeault, Les transformations de l'agriculture au Saguenay, 1852-1971 (Chicoutimi, thèse de maîtrise (études régionales), Université du Québec à Chicoutimi, 1988), 256 p.

19 Toute une série de facteurs peuvent expliquer la difficulté à retracer les occupants sur chacune des deux listes. La raison principale est sans doute l'occupation de lots déclarés dans le recensement mais qui ne furent pas concédés officiellement par le gouvernement, du moins pas avant 1872. À titre d'exemple, une note des responsables du recensement dans le manuscrit de 1861 indique pour le canton Chicoutimi: «Dans la plupart des cas, les occupants de lots n'ont pu donner les numéros des lots, ne les connaissant pas.» En outre, la période écoulée entre la concession de la terre et l'année du recensement doit également être prise en considération. Plus l'écart entre les deux dates est long, plus grandes sont les chances de voir intervenir des facteurs comme la vente, l'achat ou la transmission de la terre de même que la migration ou le décès de l'occupant. De plus, il arrive que dans le recensement le nom d'un occupant soit associé à un groupe d'individus au moment de la concession sans que nous ne puissions ultérieurement déterminer les superficies attribuables à chacun. Enfin, il y a tous les problèmes inhérents aux listes nominatives: écriture difficile (sur les manuscrits du recensement), homonymie ou substitution patronymique. Ces facteurs réunis peuvent expliquer le faible résultat du «jumelage» de notre échantillon. 


\section{LES UNITÉS DE VOLUME}

Les principales mesures de volume mentionnées dans les recensements publiés et manuscrits sont le minot et le boisseau. Le minot est une ancienne unité de mesure française. Comme l'arpent, c'est la mesure généralement en usage parmi la population francophone, mais particulièrement dans l'ancienne zone seigneuriale du Québec ${ }^{20}$. Le boisseau, mesure anglaise, serait quant à lui répandu dans les districts anglophones de la province ou dans les cantons (townships) ${ }^{21}$.

Nous avons d'abord calculé le coefficient utilisé par les responsables du recensement de 1871 pour la conversion des unités de volume, entre les données manuscrites et les données publiées. Pour chacun des quatre sous-districts Laterrière, Jonquière, Tremblay et Hébertville, nous avons mesuré les conversions appliquées aux productions de blé, d'orge et d'avoine. Le facteur utilisé était de 1,0876 boisseau par minot, soit une mesure qui se situe entre le boisseau de Winchester et le boisseau Imperial. Ainsi, s'il devait y avoir conversion des unités de volume, les facteurs devraient être de 1,107 boisseau en 1852 et vraisemblablement en $1861^{22}$, de 1,0876 en 1871 et en 1881 , et probablement de 1,073 boisseau en 1891, à cause du coefficient utilisé en 1901. Par ailleurs, l'écart entre chacun de ces trois facteurs ne dépasse pas 3,1\%.

Contrairement aux mesures de superficie pour lesquelles nous pouvions contrôler le choix des recenseurs à l'aide d'autres sources, la démonstration relative aux unités de volume pose de grandes difficultés. Au dix-neuvième siècle particulièrement, les estimations des volumes de production sont souvent approximatives. La variabilité qui en résulte, doublée d'autres impondérables susceptibles d'affecter les rendements (climat, qualité des sols, développement des techniques, etc.), ne permet pas d'extrapoler au point d'établir un nombre moyen de boisseaux à l'acre pour une certaine culture. Une solution possible consisterait à ajuster les unités de volume en fonction des modifications que nous avons apportées aux superficies. On considérerait alors que les quantités sont données en minots là où les superficies sont données en arpents, et en boisseaux dans les sous-districts où l'acre est la mesure en usage. Cependant, certains indices commandent ici une très grande prudence. Ils suggèrent même, du moins pour le Saguenay, que les

20 Deux sortes de boisseaux étaient en usage au Canada au dix-neuvième siècle. Le plus ancien est le boisseau de Winchester ( 1 minot $=1107$ boisseau). C'est la mesure utilisée par F. Lewis et R. M. McInnis pour la conversion des volumes de production du recensement de 1852. L'autre mesure est le boisseau Imperial ( 1 minot $=1073$ boisseau; elle est utilisée notamment pour la conversion des productions dans le recensement de 1901.

21 Normand Fortier, loc. cit.

22 Nous supposons que le boisseau de Winchester est également utilisé en 1861 puisque le boisseau Impérial fut introduit au Canada à partir de 1873 seulement. Voir Statuts du Canada, 36 Victoria, chapitre 47, articles 4 et 5 . Aussi, ibid., 273. 
mesures de production pourraient être tout à fait indépendantes du système de mesure des superficies. Cette réserve repose sur trois facteurs.

Nous croyons tout d'abord que la mesure des volumes de production, contrairement aux unités de superficie, n'est contrainte par aucun autre déterminisme que celui de la coutume. Nous nous situons bien sûr dans le contexte d'une société rurale traditionnelle. Au Saguenay, l'acre est devenu la mesure en usage à cause principalement du mode d'acquisition de la terre (le système cantonal). En l'absence d'une contrainte semblable touchant les unités de volume, le minot serait demeuré la mesure privilégiée.

Par ailleurs, les journaux saguenayens de l'époque constituent pour nous une référence historique privilégiée en fournissant une description relativement fidèle de la réalité quotidienne et, notamment, de tout ce qui se rapportait à l'agriculture. Nous les avons donc utilisés comme outil de validation qualitatif. D'une manière ponctuelle, nous avons relevé dans le Progrès du Saguenay ${ }^{23}$, pour les années 1888, 1890 et 1892 , les mentions où il était question de volumes de production en agriculture. Dans presque tous les cas, le minot est l'unité de mesure indiquée. En fait, sur une trentaine de références à des volumes de production, une seule mentionne le boisseau. Bien plus, pour les trois années que nous avons relevées, l'acre et le minot sont les mesures utilisées pour exprimer des valeurs de rendement. Il s'agit là bien sûr d'une évaluation qualitative, mais elle va précisément dans le sens de nos conclusions précédentes.

Finalement, dans son article sur l'utilisation des recensements canadiens pour l'étude de l'agriculture québécoise, N. Fortier évoque le cas de deux cantons du district de Saint-Hyacinthe (cantons d'Upton et d'Acton) pour lesquels il semble exister une similitude avec les unités de mesure en usage au Saguenay au XIXe siècle.

Dans les sous-districts des cantons d'Upton et d'Acton (...) qui comptent une population francophone à $80 \%$ au moins, les autorités du recensement (de 1871) transforment bien les minots en boisseaux mais laissent intactes les mesures de superficie, les traitant comme des acres. Il faut rappeler que les concessions originelles se font en acres dans les cantons. ${ }^{24}$

L'exemple de ces deux cantons, situés sur les marges de la seigneurie de Saint-Hyacinthe, présente beaucoup de points communs avec

23 Le Progrès du Saguenay, publication hebdomadaire, Chicoutimi, 1887-1965.

24 Ibid., 271. 
le Saguenay. On retrouve une population très majoritairement francophone en provenance des anciennes limites seigneuriales et qui se déploie à l'intérieur de nouvelles divisions cantonales. Dans le cas des cantons d'Acton et d'Upton, ce sont les responsables du recensement qui attestent l'usage simultané du minot et de l'acre, alors qu'au Saguenay nos tests de validation tendent vers la même conclusion.

À la lumière des trois indices que nous avons identifiés (la pratique coutumière, l'appui d'une source complémentaire et l'analogie avec un exemple extérieur à la région), nous croyons indiqué de considérer comme des minots les volumes de production donnés dans les recensements saguenayens du dix-neuvième siècle. Cela invite à convertir en boisseaux les volumes déclarés en minots dans les recensements de 1852,1861 et 1891 et à garder intactes les valeurs déjà converties par les responsables des recensements de 1871 et de 1881 .

\section{FACTEURS DE CONVERSION}

$\mathrm{Au}$ tableau 1, nous proposons un modèle de conversion global pour l'ensemble des unités de volume et de superficie se rapportant au Saguenay. Ce modèle serait évidemment beaucoup plus précis si nous avions pu tenir compte des caractéristiques de tous les sous-districts ou même de chacun des occupants de terre. Cependant, nous croyons nous approcher davantage de la réalité de l'époque en procédant ainsi, plutôt qu'en utilisant les valeurs décennales telles qu'elles apparaissent dans les recensements publiés et manuscrits du dix-neuvième siècle.

Par ailleurs, afin de montrer l'incidence de notre grille de conversion sur les valeurs décennales, nous donnons en exemple le rendement moyen de l'avoine pour l'ensemble de la région du Saguenay en 1852 (tableau 2).

Le modèle que nous privilégions $(\mathrm{C})$ hausse d'environ $10 \%$ le rendement moyen de l'avoine si on le compare à la valeur calculée directement à partir des données non modifiées du recensement. Il se situe par ailleurs à mi-chemin entre ce dernier et le rendement tiré d'une conversion unilatérale des superficies et des volumes de production.

\section{LES RECENSEMENTS DU XXe SIECLE}

Les valeurs des mesures de production et de superficie dans les recensements publiés du vingtième siècle posent sans doute moins de problèmes de conversion que les relevés des décennies précédentes. Bien que pour cette période notre argumentation se heurte assez rapidement à l'absence de recensements manuscrits, un certain nombre d'indices tendent à confirmer cette hypothèse. D'une part, à partir de 1901, on n'évoque dans aucun recensement la nécessité de convertir 
TABLEAU 1

Règles à suivre pour l'utilisation des unités de mesure des recensements canadiens pour les divisions du Saguenay

\begin{tabular}{|c|c|c|c|c|}
\hline & \multicolumn{2}{|c|}{ Recensements publiés } & \multicolumn{2}{|c|}{ Rencensements manuscrits } \\
\hline ANNEES & SUPERFICIES & VOLUMES & SUPERFICIES & VOLUMES \\
\hline 1852 & $\begin{array}{l}\text { sans } \\
\text { conversion }\end{array}$ & $\begin{array}{l}\text { conversion } \\
(\times 1.107)\end{array}$ & $\begin{array}{l}\text { sans } \\
\text { conversion; } \\
\text { en exprimant } \\
\text { les données } \\
\text { en acres }\end{array}$ & $\begin{array}{l}\text { conversion } \\
(\times 1.107)\end{array}$ \\
\hline 1861 & $\begin{array}{l}\text { sans } \\
\text { conversion }\end{array}$ & $\begin{array}{l}\text { conversion } \\
(\times 1.107)\end{array}$ & $\begin{array}{l}\text { sans } \\
\text { conversion; } \\
\text { en exprimant } \\
\text { les données } \\
\text { en acres }\end{array}$ & $\begin{array}{l}\text { conversion } \\
(\times 1.107)\end{array}$ \\
\hline 1871 & $\begin{array}{l}\text { conversion } \\
(\times 1.1834)\end{array}$ & $\begin{array}{l}\text { sans } \\
\text { conversion: } \\
\text { (déjà converti } \\
\text { par } \times 1.0876 \text { ) }\end{array}$ & $\begin{array}{l}\text { sans } \\
\text { conversion: } \\
\text { en exprimant } \\
\text { les données } \\
\text { en acres }\end{array}$ & $\begin{array}{l}\text { conversion } \\
(\times 1.0876)\end{array}$ \\
\hline 1881 & $\begin{array}{l}\text { conversion } \\
(\times 1.1834)\end{array}$ & $\begin{array}{l}\text { sans } \\
\text { conversion; } \\
\text { (déjà converti } \\
\text { par × 1.0876) }\end{array}$ & $\begin{array}{l}\text { données } \\
\text { non } \\
\text { disponibles }\end{array}$ & $\begin{array}{l}\text { données } \\
\text { non } \\
\text { disponibles }\end{array}$ \\
\hline 1891 & $\begin{array}{l}\text { sans } \\
\text { conversion }\end{array}$ & $\begin{array}{l}\text { conversion } \\
(\times 1.073)\end{array}$ & $\begin{array}{l}\text { données } \\
\text { non } \\
\text { disponibles }\end{array}$ & $\begin{array}{l}\text { données } \\
\text { non } \\
\text { disponibles }\end{array}$ \\
\hline
\end{tabular}

TABLEAU 2

Exemple d'application des règles de conversion: le rendement de l'avoine en 1852 au Saguenay selon trois méthodes de calcul.

\begin{tabular}{|c|c|c|c|}
\hline \multicolumn{1}{|c|}{ MÉTHODES } & $\begin{array}{c}\text { SUPERFICIES } \\
\text { (acres) }\end{array}$ & $\begin{array}{c}\text { VOLUMES } \\
\text { (boisseaux) }\end{array}$ & $\begin{array}{c}\text { RENDEMENTS } \\
\text { (boisseaux/acres) }\end{array}$ \\
\hline $\begin{array}{c}\text { A. Données tirées du } \\
\text { recensement publié }\end{array}$ & 1151 & 7325 & $\mathbf{6 . 4}$ \\
\hline $\begin{array}{l}\text { B. Double conversion } \\
\text { (comme s'il } \\
\text { s'agissait de } \\
\text { mesures françaises }\end{array}$ & $\begin{array}{c}973 \\
\text { (ou } 1151 \\
\text { arpents } \\
\times 0,845)\end{array}$ & $\begin{array}{c}8109 \\
\text { (ou 7325 } \\
\text { minots } \\
\times 1,107)\end{array}$ & $\mathbf{8 . 3}$ \\
\hline $\begin{array}{l}\text { C. Conversion des } \\
\text { minots seulement } \\
\text { (modèle privilégié } \\
\text { par l'auteur) }\end{array}$ & 1151 & $\begin{array}{c}8109 \\
(\text { ou } 7325 \\
\text { minots } \\
\times 1,107)\end{array}$ & $\mathbf{7 . 1}$ \\
\hline
\end{tabular}


les unités françaises de certains districts en mesures anglaises ${ }^{25}$. D'autre part, avec les changements introduits dans le mode de relevé, on est en droit de s'attendre à une rigueur accrue dans la collecte et le traitement des données. C'est ce que suggère par exemple une note dans le questionnaire du recensement de 1911.

Si le renseignement est donné en arpent, le recenseur écrira le mot arpent au-dessus du chiffre qui indique la superficie, et les entrées dans les autres tableaux agricoles qui se rapportent à cette même personne devront être basées sur la même mesure. ${ }^{26}$

Enfin, nous croyons que l'introduction des mesures anglaises dans l'agriculture (principalement celles se rapportant à la région du Saguenay) se fit graduellement avec la disparition de l'agriculture d'autosubsistance. En effet, le besoin croissant d'alimenter un marché en grande partie extra-régional a nécessairement favorisé une standardisation des unités de mesure à l'échelle du pays. Pour toutes ces raisons, nous préférons conserver les valeurs des unités de mesure telles qu'elles sont données dans les recensements publiés du vingtième siècle.

\footnotetext{
1891.

5 On le fait bien sûr en 1901, mais pour souligner l'erreur commise au recensement de

26 Recensement canadien, 1911, volume IV: 425.
} 